\title{
Metagenomics analysis using next generation sequencing of vaginal samples from community practices in the us
}

\begin{abstract}
Molecular-based Phylogenetic analyses, including the newly-completed Human Microbiomes Project and other next-generation sequencing (NGS) studies have revealed the complexity of the vaginal micro biome in health and disease. The healthy vagina tends to be dominated by hydrogen peroxide and lactic acid producing organisms, the most important of which are Lactobacillus species. The loss of these organisms and the rise of non-resident microorganisms can lead to a diseased state such as bacterial vaginosis (BV or vaginal bacteriosis). The new consensus generated by these studies is that BV is not a single disorder or caused by one etiological agent but rather it is a spectrum of symptoms clinically characterized by vaginal discharge, increased $\mathrm{pH}$ and malodor caused by an imbalance in the normal vaginal bacterial flora. NGS techniques allow for the capture of the entire vaginal micro biome in a single assay and create the ability not only to obtain a better understanding of the causes of BV but also lead to a viable clinical method for detecting it. In this study, we use next generation sequencing of $16 \mathrm{~S}$ rRNA genes to obtain the vaginal micro biome from 270 vaginal swabs. Our analysis includes an in-depth characterization of bacterial communities identified, including quantitation and composition of the communities present in normal and BV samples. Our results show vaginal samples can be broken down into three distinct groups that segregate largely based on degree and type of Lactobacillus content and the presence or absence of $G$. vaginalis
\end{abstract}

Volume 2 Issue 2 - 2015

\section{William Budd T, Michael Harwich, David G Bostwick, Greg Meyers, Jeri Dilts, Katherine O'Hanlon, John Woody, Letitia Mason, Thomas Reynolds}

Department of Health and Human Services, American International Biotechnology, USA

Correspondence: Thomas Reynolds, Department of Health and Human Services, American International Biotechnology, 601 Biotech Drive, Richmond,VA 23235, USA, Tel8043057648, Email treynolds@aibiotech.com

Received: February 27, 2015 | Published: March 24, 2015

Keywords: metagenomics, bacterial vaginosis, next generation sequencing, gardnerella vaginalis, 16 s sequencing

Abbreviations: ATCC, american type culture collection; $\mathrm{BHI}$, brain heart infusion; BV, bacterial vaginosis; CLIA, clinical laboratory improvements amendment; DNA, deoxyribonucleic acid; $\mathrm{H}_{2} \mathrm{O}_{2}$, hydrogen peroxide; $\mathrm{LB}$, lutria broth; $\mathrm{NaCl}$, sodium chloride; NGS, next generation sequencing; OD, optical density; PERL, pattern extraction and reporting language; PGM, personal genome machine; PID, pelvic inflammatory disease; rRNA, ribosomal ribonucleic acid; Spp, species; TSA, tryptic soy agar

\section{Introduction}

Recent estimates from the human micro biome project estimate that the ratio of bacteria to human cells is 10:1. ${ }^{1}$ Evidence continues to grow suggesting that the composition and function of the micro biome is important for normal human development and continued homeostasis. Microorganisms tend to colonize at various sites and form complex communities adapted to their specific environment. ${ }^{2}$ The vagina tends to be dominated by hydrogen peroxide and lactic acid producing organisms, the most important of which are Lactobacillus species. $^{3}$ The loss of these organisms and the rise of non-resident microorganisms can lead to a diseased state such as bacterial vaginosis (BV, or vaginal bacteriosis). BV occurs when healthy lactobacilli are replaced with a diverse polymicrobial population comprised of multiple taxa, including commensal facultative anaerobes and other pathogenic bacteria. ${ }^{4,5}$ Clinically $\mathrm{BV}$ is characterized by a spectrum of symptoms including vaginal discharge, increased $\mathrm{pH}$, and malodor.

$\mathrm{BV}$ is the most commonly diagnosed vaginal infection in women of child-bearing age, accounting for $27-41 \%$ prevalence in Americans. ${ }^{6,7}$
It is common in postmenopausal women, with a prevalence of $23 \%$ that increases with age. ${ }^{8} \mathrm{BV}$ is an important medical condition associated with more serious adverse outcomes such as acquisition of sexually transmitted infections/ HIV, pre-term child birth, and development of pelvic inflammatory disease (PID). ${ }^{9}$ Activities associated with an increased risk of BV include an increased number of male sex partners ( $>3$ in 12months), having sex with a female in the previous 12 months, previous pregnancies and cigarette smoking. ${ }^{10}$ Protected sex using a condom is considered a protective behavior and has been shown to limit the incidence of $\mathrm{BV}$.

One of the greatest clinical challenges with BV is dealing with an extremely high rate of treatment failure $(41 \%$ to $67 \%$ within months) despite use of oral or intravaginal antibiotics. Treatment failure is frustrating to patients and clinicians. ${ }^{11-13}$ There exist conflicting theories regarding the exact etiology of treatment failure. Some clinicians theorize that BV recurrence is a relapse of the same infection, while others believe it is not relapse but rather each occurrence is a new infection. Contributory risk factors for treatment failure include unprotected sex, sex with partners colonized by BVassociated bacteria, repeated bouts of $\mathrm{BV}$, and use of an intrauterine device. As the frequency of BV increases with the number of sexual partners and unprotected sex, it was hypothesized that BV may be a sexually transmitted infection supporting the concept of reinfection. ${ }^{14}$ However, treatment of an infected woman's partner did not statistically reduce the incidence of BV recurrence. Evidence suggests that BV recurrence is a relapse of the primary infection because there is a failure of vaginal re-colonization with healthy 
Lactobacillus spp. and continued colonization by anaerobic bacteria. Some of the BV-associated anaerobes, like Gardnerella vaginalis grow as a tenacious biofilm that adheres to the vaginal epithelium, making successful eradication more difficult. ${ }^{11}$ Several of the anaerobic bacteria associated with $\mathrm{BV}$, such as $G$. vaginalis and $M$. curtisii, have low in vitro susceptibility to metronidazole, the drug most commonly prescribed to treat BV contributing to relapse if not all of the anaerobes are eradicated. ${ }^{15}$

The etiologic enigma has caused physicians and clinicians to diagnose BV based upon clinical criteria rather than on cultivation based tests and other standard microbial tests used when one organism is suspect. One such method for diagnosis of BV is through the use of Amsel's criteria, where at least three of the following four conditions must be met: presence of a thin, homogenous discharge; vaginal fluid $\mathrm{pH}$ greater than 4.5; positive whiff test (generation of fishy odor when $10 \%$ potassium hydroxide is added to vaginal fluid); and microscopic presence of clue cells (sloughed epithelial cells coated with adherent bacteria). ${ }^{16}$ Amsel's criteria cannot identify the etiologic agents of infection. A second clinical method for diagnosing BV is through use of the Nugent's score which attempts to distinguish normal vaginal flora (lactobacilli are typically gram-positive rods) from BV-associated organisms (gram-negative organisms) by performing Gram's stain on vaginal fluid. ${ }^{17}$ Neither of these methods is perfect or absolute; both tend to be highly subjective and depend on the skill and experience of the clinician processing the sample. ${ }^{18}$ Additionally, use of the Nugent score is based on an assumption of what the normal flora should be, which might not be totally accurate or complete.

Molecular-based phylogenetic analyses using 16S ribosomal RNA (rRNA) gene sequencing, including the newly-completed Human Microbiome Project and other next-generation sequencing (NGS) studies have revealed the complexity of the vaginal microbiome in health and disease. ${ }^{1,2,19}$ The 16s rRNA gene is an essential component of prokaryotic translation machinery. It is highly conserved and is a useful genetic target for microbiome type studies. The 16S rRNA molecule features areas of sequence conservation that can be used for primer design allowing for amplification of this target from various genetic backgrounds, yet there is also enough sequence diversity present to allow for the ability to identify organisms down to the genera and species level. ${ }^{20}$ Semiconductor sequencing technology (Ion Torrent) reduces the cost of per base sequencing and has been shown to be amenable to clinically relevant samples. ${ }^{21}$ Using a semiconductor sequencer, for the first time we can deliver clinically actionable results in time for a physician to make treatment decisions.

In addition to identification of resident bacterial species, $16 \mathrm{~S}$ ribosomal RNA gene sequencing using next generation sequencing (NGS) technology allows numerous improvements compared with existing methods of detection. Currently, clinical assays are capable of qualitative identification of bacterial species present in a complex community. It is known that many women are colonized with $G$. vaginalis but do not suffer symptoms of BV. ${ }^{18,19}$ Quantification of microbial community constituency will aid clinicians to more accurately assess the total microbiome of their patient, identifying those that are in need of treatment. Another benefit of an NGS based assay, is other gene targets can easily be easily added to an NGS panel. Virulence factors such as sialidase could enhance bacterial survival, invasion and/or tissue destruction; resistance genes can confer a selective advantage to bacteria making them immune to common treatments. The addition of these virulence factors and resistance genes can aid in diagnosis and treatment planning. ${ }^{22,23}$ In this study, we evaluated the composition of the vaginal microbiome by in-depth next generation sequencing of 270 women from community GYN practices in the United States. Our goal for this study is to better define the microbial communities inherent to normal and BV states, identify causative/ complementary bacterial communities, and show that vaginal microbiome analysis is a viable approach to the detection of BV in clinical samples.

\section{Materials and methods}

\section{Ethics statement}

The data used in this study came from samples submitted to a CLIA approved clinical lab and was not collected specifically for this study. Researchers involved in this study were not aware of the participant's identity, health status, or other personal information.

\section{Media preparation}

Brain Heart Infusion (BHI) broth (BD, Franklin Lakes, NJ), de Man Rogosa and Sharpe (MRS) broth (BD, Franklin Lakes, NJ), and Luria Bertani (LB) broth made according to the manufacturer's recommendations unless otherwise noted. Corresponding solid media were also prepared by the addition of agar to $7.5 \%$. Nutrient broth (BD, Franklin Lakes, NJ) and agar were prepared supplemented with 3\% NaCl. Tryptic Soy Agar (TSA) + 5\% sheep's blood (BD, Franklin Lakes, NJ), CDC anaerobe blood agar (BD, Franklin Lakes, NJ), Human bi-layer tween (Remel, Lenexa, KS), Brucella blood agar (Hardy Diagnostics, Santa Maria, CA) and Cooked Meat Media (Hardy Diagnostics, Santa Maria, CA) were purchased ready to use from the indicated companies.

\section{Bacterial growth conditions}

Bacterial and fungal species (Table 1) were ordered from the American Type Culture Collection (ATCC) and grown in the indicated solid and liquid media. Cultivation of some anaerobes were routinely performed in BHI broth supplemented with $0.1 \%$ glucose (ACROS Organics Geel, Belgium), 0.1\% starch (Spectrum, New Brunswick, $\mathrm{NJ}$ ), 2\% gelatin (G Biosciences, St. Louis, $\mathrm{MO}$ ) and $1 \%$ yeast extract (BD, Franklin Lakes, NJ) (sBHI). All cultures were incubated at $37^{\circ} \mathrm{C}$. Anaerobic conditions were generated by the GasPak EZ Anaerobe Container System (BD, Franklin Lakes, NJ) system. Organisms requiring $\mathrm{CO}_{2}(5 \%)$ for growth, were cultured in $\mathrm{CO}_{2}$ incubator (VWR Symphony). Liquid cultures were incubated until visible turbidity was detected. All incubations were carried out at $37^{\circ} \mathrm{C}$.

Table I Organisms cultivated and the appropriate media and growth conditions

\begin{tabular}{llll}
\hline Organism & $\begin{array}{l}\text { Solid } \\
\text { media }\end{array}$ & $\begin{array}{l}\text { Liquid } \\
\text { media }\end{array}$ & $\begin{array}{l}\text { Culture } \\
\text { conditions }\end{array}$ \\
\hline Gardnerella vaginalis & Blood & $\mathrm{sBHI}$ & Anaerobic \\
Atopobium vaginae & Blood & $\mathrm{sBHI}$ & Anaerobic \\
Lactobacillus crispatus & $\mathrm{MRS}$ & $\mathrm{MRS}$ & $5 \% \mathrm{CO}_{2}$ \\
Lactobacillus iners & $\mathrm{HBT}$ & $\mathrm{sBHI}$ & $5 \% \mathrm{CO}_{2}$ \\
Streptococcus agalactiae & $\mathrm{BHI}$ & $\mathrm{BHI}$ & Aerobic \\
Bacteroides fragilis & $\mathrm{Blood}$ & $\mathrm{sBHI}$ & Anaerobic \\
Prevotella bivia & $\mathrm{Blood}$ & $\mathrm{sBHI}$ & Anaerobic \\
Clostridium septicum & Blood & $\mathrm{sBHI}$ & Anaerobic \\
\hline
\end{tabular}


Table Continued....

\begin{tabular}{llll}
\hline Organism & $\begin{array}{l}\text { Solid } \\
\text { media }\end{array}$ & $\begin{array}{l}\text { Liquid } \\
\text { media }\end{array}$ & $\begin{array}{l}\text { Culture } \\
\text { conditions }\end{array}$ \\
\hline Fusobacterium nucleatum & Blood & $\mathrm{sBHI}$ & Anaerobic \\
Veillonella parvula & Blood & $\mathrm{sBHI}$ & Anaerobic \\
Enterococcus faecalis & $\mathrm{BHI}$ & $\mathrm{BHI}$ & Aerobic \\
Candida albicans & $\mathrm{SBA}$ & $\mathrm{LB}$ & Aerobic \\
Neisseria meningitidis & $\mathrm{BHI}$ & $\mathrm{BHI}$ & $5 \% \mathrm{CO}_{2}$ \\
Staphylococcus aureus & $\mathrm{BHI}$ & $\mathrm{BHI}$ & Aerobic \\
Pseudomonas aeruginosa & $\mathrm{LB}$ & $\mathrm{LB}$ & Aerobic \\
Escherichia coli & $\mathrm{LB}$ & $\mathrm{LB}$ & Aerobic \\
\hline
\end{tabular}

Blood agar - Brucella, CDC Anaerobe, or TSA II media were used.

\section{Bacterial enumeration and genomic DNA isolation}

Once plank tonic bacterial growth had been achieved, an aliquot of each culture was removed to measure the Optical Density at $600 \mathrm{~nm}$ $\left(\mathrm{OD}_{600}\right)$. Liquid cultures were then serially diluted and plated onto appropriate solid media to enumerate the viable cells in each. Aliquots of each culture were also enumerated for total cells with a Z1 Particle Counter (Beckman-Coulter, Brea, CA). Aliquots of these cultures were used to isolate genomic DNA from each bacterial species via the DNeasy blood and tissue kit (Qiagen Valencia, CA). Isolated DNA was quantitated on a NanoDrop 8000 Spectrophotometer. A genome copy calculator from Thermo Scientific was used to ensure that $10^{9}$ total copies $/ \mathrm{mL}$ were used for each mock sample.

\section{Clinical study participants}

We undertook a retrospective diagnostic cross-sectional study with consecutive recruitment of all next-generation sequencing (NGS) vaginal swab specimens examined at American International Biotechnology LLC (AIBioTech, Richmond, VA) between July 1, 2014 and November 30, 2014. Vaginal swabs were collected from 270 women from community gynecology practices in the United States. All study participants consented to participate. All samples were deidentified to researchers involved in this study in order to maintain patient confidentiality.

\section{DNA Extraction}

Cervical and Vaginal swab specimens were collected and stored using the $\mathrm{BD}^{\mathrm{TM}}$ Universal Viral Transport System (Becton Dickinson, $\mathrm{MD}$ ) according to the manufacturer's instructions. Extraction of DNA was performed using the RTP Bacteria DNA Mini Kit (STRATEC Molecular, Berlin) according to the manufacturer's instructions. Briefly, the sample is mixed and $400 \mu l$ is transferred to the provided extraction tube. $400 \mu \mathrm{l}$ Re-suspension Buffer added, incubated for $10 \mathrm{~min}$ at $37^{\circ} \mathrm{C}$ in a thermo mixer, then for $10 \mathrm{~min}$ at $65^{\circ} \mathrm{C}$ in a thermo mixer, then for $5-10$ minutes at $95^{\circ} \mathrm{C}$ in a thermo mixer. $400 \mu 1$ Binding Buffer B6 is added, vortexed, and transferred to RTA-Spin Filter. The column is washed twice with the provided wash buffers. To eliminate residual ethanol, samples are centrifuged for $4 \mathrm{~min}$ at maximum speed. Samples are eluted with $200 \mu$ lof the provided elution buffer at $65^{\circ} \mathrm{C}$. Samples are stored at $4^{\circ} \mathrm{C}$ for up to one month or stored at $-20^{\circ} \mathrm{C}$.

\section{Semi-conductor based next generation sequencing}

Universal primers were designed to amplify multiple regions of the 16S rRNA gene sequences. DNA libraries were generated using the Ion AmpliSeq ${ }^{\mathrm{TM}}$ Library Kit 2.0-96LV (Cat. no. 4480441). See Ion AmpliSeqTM Library Preparation (Publication Part Number MAN0006735 Revision A.0). Two separate primer pools were utilized in the initial amplification reaction. The pools were combined before proceeding with the FuPa reaction. Ion Xpress ${ }^{\mathrm{TM}}$ Barcode Adapters 1-16 Kit (Cat. No. 4471250) and Barcode Adapters 1732 Kit (Cat. No. 4474009) were utilized for sample barcoding and adaptor ligation. AmPureAgencourt XP (Cat. No. A63882, Beckman) magnetic PCR cleanup beads were utilized as indicated in the Ion AmpliSeq ${ }^{\mathrm{TM}}$ Library Preparation User Guide. Sample libraries were normalized by Ion Library Equalizer ${ }^{\mathrm{TM}}$ Kit (Cat. no. 4482298), yielding a final sample library of $100 \mu \mathrm{L}$ at $100 \mathrm{pM}$ concentration. Emulsion PCR (emPCR) was performed on the Ion One Touch ${ }^{\mathrm{TM}} 2$ Instrument (Cat. No. 4474778) as indicated in Ion PGM ${ }^{\mathrm{TM}}$ Template OT2 200 Kit (Publication Number MAN0007220. Revision 5.0). Reagent kit Ion PGM ${ }^{\mathrm{TM}}$ Template OT2 200Kit (Cat. no. 4480974). Briefly, normalized 100pM sample libraries were pooled and loaded with OT2 kit reagents with ISP beads. Samples were processed for enrichment on the Ion One Touch ${ }^{\mathrm{TM}}$ ES no later than 16hours post emPCR run completion. Enrichment of ISPs was achieved using Reagent kit Ion PGM ${ }^{\mathrm{TM}}$ Template OT2 200 Kit (Cat. no. 4480974) and DynaBeadsMyOne streptavidin C1 beads (cat. no. 65001, Life Technologies) according to the manufacturer's protocols (Ion PGM ${ }^{\mathrm{TM}}$ Template OT2 200 Kit User Guide). Ion Torrent Ion $316^{\mathrm{TM}}$ Chip Kit v2 (Cat. No. 4483188, Life Technologies) were prepared and loaded according to the manufacturer's recommendation (Ion PGM ${ }^{\mathrm{TM}}$ Sequencing 200Kit v2 (Publication Number MAN0007273 Revision 3.0). Chips were manually loaded with enriched ISPs with primed sequencing polymerase (provided in kit) using Rainin ${ }^{\circledR}$ pipette tips SR-L200F. The Ion Torrent PGM was run according to Ion Torrent 316 chip specifications, 500 flows, and use of $18 \mathrm{M} \Omega$ water system (multistage system including: Carbon tank, RO membrane, UV irradiation, post-filtering, deionization, and an Elga water polisher). Standard compressed nitrogen gas supplied to the PGM system.

\section{Analysis of next generation sequencing data}

A reference $16 \mathrm{~S}$ rDNA non-redundant, database was compiled for organisms likely to be present in the vagina based on data from the Vaginal Microbiome Consortium. ${ }^{20}$ This resource is based upon an in-depth analysis of over 1000 participants. Full 16S sequences were compiled for over 1,300 bacteria and used for comparison of targeted sequencing data. A custom bioinformatics solution was created to process the data using PERL. Reads greater than 75 nucleotides in length were used to align to a manually curated USEARCH database of the organisms described. ${ }^{24}$ In order to be considered as detected, the organism had to exceed a threshold of 100 reads across multiple variable regions of the $16 \mathrm{~S}$ rDNA molecule. Each detected organism was approximated by dividing the number of reads mapped to the organism by the total number of reads mapped to all bacteria. Appropriate controls were included in each sequencing run including a positive control composed of multiple bacteria in known proportions, an extraction blank and a non-template library blank. Acceptance of the sequencing results required that the positive control return each bacterium in the mix, and less than 7500 reads with no organism calls for both blanks.

\section{Statistical analysis}

All statistical analyses were performed using $\mathrm{R}$ Project for Statistical Computing (http://www.r-project.org). ${ }^{25}$ A Kruskal-Wallis non-parametric analysis of variance was used to compare distributions 
between groups. All box plots were created using R. Microsoft Excel was used to create the stacked bar plots in (Figure $4 \& 6$ ).

\section{Results and discussions}

\section{Mock Communities}

Species classification of organisms within a complex microbial community is inherently difficult due to the ubiquitous nature of the 16S rRNA molecule. Multiple mock communities were created

Table 2 Mock communities containing bacteria in contrasting proportions

\begin{tabular}{|c|c|c|c|}
\hline $\begin{array}{l}\text { Lactobacillus crispatus } \\
\text { genome copies }\end{array}$ & Other organism & $\begin{array}{l}\text { Other bacteria } \\
\text { genome copies }\end{array}$ & Both detected \\
\hline $1.90 \times 10^{6}$ & Lactobacillus iners & $1.90 \times 10^{5}$ & Yes \\
\hline $1.90 \times 10^{6}$ & Gardnerella vaginalis & $1.90 \times 10^{5}$ & Yes \\
\hline $1.90 \times 10^{6}$ & Fusobacterium nucleatum & $1.90 \times 10^{5}$ & Yes \\
\hline $1.90 \times 10^{6}$ & Veillonella parvula & $1.90 \times 10^{5}$ & Yes \\
\hline $1.90 \times 10^{6}$ & Streptococcus agalactiae & $1.90 \times 10^{5}$ & Yes \\
\hline $1.90 \times 10^{6}$ & Prevotella bivia & $1.90 \times 10^{5}$ & Yes \\
\hline $1.90 \times 10^{6}$ & Atopobium vaginae & $1.90 \times 10^{5}$ & Yes \\
\hline $1.90 \times 10^{6}$ & Bacteroides fragilis & $1.90 \times 10^{5}$ & Yes \\
\hline $1.90 \times 10^{6}$ & Clostridium septicum & $1.90 \times 10^{5}$ & Yes \\
\hline $1.90 \times 10^{6}$ & Enterococcus faecalis & $1.90 \times 10^{5}$ & Yes \\
\hline $1.90 \times 10^{6}$ & Neisseria meningitis & $1.90 \times 10^{5}$ & Yes \\
\hline $1.90 \times 10^{6}$ & Staphylococcus aureus & $1.90 \times 10^{5}$ & Yes \\
\hline $1.90 \times 10^{6}$ & Pseudomonas aeruginosa & $1.90 \times 10^{5}$ & Yes \\
\hline $1.90 \times 10^{6}$ & Escherichia coli & $1.90 \times 10^{5}$ & No* \\
\hline
\end{tabular}

*Escherichia coli was incorrectly called as Shigella spp.

The vagina is a complex environment which can be inhabited by multiple bacterial species as well as a number of fungal organisms. Vulvovaginal candidiasis occurs in $75 \%$ of women over $25 y$ ears of age. Candida albicans is the most common causative agent of yeast infections worldwide. It is not uncommon for a woman to have concomitant bacterial vaginosis and Vulvovaginal candidiasis. A clinical assay to diagnose BV must be able to accurately distinguish these syndromes from one another. To ensure that our assay could detect BV related organisms in the presence of $C$. albicans, three unique mock communities were created containing Candida albicans along with various bacterial species related to BV. Each mock sample contained a unique mixture of bacterial genomes and a varying number of $C$. albicans genome copies (Table 3B-3D). Bacteria were amplified and sequenced in the presence of C. albicans (Table 3A), likewise $C$. albicans was amplified and sequenced in the presence of the bacterial components. Further, each bacterium in the Candidia mixes was correctly identified at the species level. Analysis of the mock communities demonstrated that this next generation sequencing approach was capable of correctly identifying members of a complex bacterial and fungal community to the species level and within a milieu of proportions that varied in several orders of magnitude. These mock communities established the validity of the assay. to evaluate the ability to detect bacterial species commonly found in the vagina. The healthy vaginal flora is dominated by Lactobacillus crispatus. Multiple mock samples were created to test the ability to detect organisms found in vaginal samples in the presence of a higher number of L. crispatus genome copies (Table 2). With one exception, the organisms evaluated were positively classified at the species level. E. coli was incorrectly called as a Shigella species. Many of the organisms included in the mock community testing are important as they are suspected to be involved in the pathogenesis of bacterial vaginosis.
Table 3 Mock Communities Containing Bacteria and Candida albicans Table 3A

\begin{tabular}{lllll}
\hline Sample ID & $\begin{array}{l}\text { Bacteria } \\
\text { genome } \\
\text { copies }\end{array}$ & $\begin{array}{l}\text { Candida albicans } \\
\text { genome copies }\end{array}$ & $\begin{array}{l}\text { Bacteria } \\
\text { detected }\end{array}$ & $\begin{array}{l}\text { Candida } \\
\text { albicans } \\
\text { detected }\end{array}$ \\
\hline CVI & $5.0 \times 10^{8}$ & $1.0 \times 10^{6}$ & Yes & Yes \\
CV2 & $6.0 \times 10^{8}$ & $8.0 \times 10^{5}$ & Yes & Yes \\
CV3 & $7.5 \times 10^{8}$ & $5.0 \times 10^{5}$ & Yes & Yes \\
\hline
\end{tabular}

\section{Table 3B}

\begin{tabular}{lll}
\hline Bacteria expected in mix CVI & $\begin{array}{l}\text { Genome } \\
\text { copies }\end{array}$ & $\begin{array}{l}\text { Bacteria } \\
\text { found }\end{array}$ \\
\hline Lactobacillus crispatus & 100000 & Yes \\
Lactobacillus iners & 100000 & Yes \\
Streptococcus agalactiae & 400000 & Yes \\
Enterococcus faecalis & 100000 & Yes \\
Staphylococcus aureus & 200000 & Yes \\
Pseudomonas aeruginosa & 100000 & Yes
\end{tabular}


Table 3C

\begin{tabular}{lll}
\hline Bacteria expected in mix CV2 & $\begin{array}{l}\text { Genome } \\
\text { copies }\end{array}$ & $\begin{array}{l}\text { Bacteria } \\
\text { found }\end{array}$ \\
\hline Lactobacillus iners & 200000 & Yes \\
Gardnerella vaginalis & 300000 & Yes \\
Prevotella bivia & 600000 & Yes \\
Atopobium vaginae & 200000 & Yes \\
Bacteroides fragilis & 100000 & Yes \\
Neisseria gonorrhoeae & 100000 & Yes
\end{tabular}

Table 3D

\begin{tabular}{lll}
\hline Bacteria expected in mix CV3 & Genome copies & Bacteria found \\
\hline Fusobacterium nucleatum & 200000 & Yes \\
Veillonella parvula & 300000 & Yes \\
Staphylococcus aureus & 200000 & Yes
\end{tabular}

\section{Clinical samples}

To our knowledge, this is the first clinical application of a next generation sequencing assay for diagnosis of BV. This application has only recently become possible due to reductions in sequencing cost and increases in throughput. The cost to consumer of this assay is similar to that of other molecular diagnostic assays used to test for BV. In order for a clinical assay to provide clinically meaningful data, it must be returned to the physician in a meaningful period of time. Mean turnaround time for this assay was 3.7 days from sample receipt to final written report to clinicians. With this rapid turnaround, for the first time clinicians are able to receive clinically actionable information that shows a more comprehensive picture of the vaginal microbiome than any single target stand-alone test can provide. Armed with these results, clinicians can accurately assess the entirety of the vaginal flora.

After collection in the clinic, vaginal or cervical swabs $(n=270)$ were sent by overnight delivery to the lab for processing and DNA extracted within 24hours of receipt. Average age of participants in this study was 36years $(\mathrm{SD}=14, \min =18, \max =90)$. The mean number of reads sequenced in each sample was 153,545 . In the samples collected, a large number of diverse organisms were found (Table 4). The most common potential pathogen was Gardnerella vaginalis found in $\sim 65 \%$ of cases. Until recently, G. vaginalis was considered the sole causative agent of bacterial vaginosis. Current evidence challenges this hypothesis as G. vaginalis may be present in up to $60 \%$ of women that do not have symptoms of BV. ${ }^{26}$ In a paper published in 1955 , Gardner and Dukes inoculated 13 healthy women with pure cultures of $G$. vaginalis and only one developed any symptoms associated with $\mathrm{BV} .{ }^{27}$ It appears other factors are involved in the pathogenesis in at least some cases of BV. Analysis of the microbial community revealed that the number of unique bacterial genera significantly differs depending upon the presence or absence of Gardnerella vaginalis (Figure 1A). When $G$. vaginalis is present, there tended to be a larger number of bacterial genera present ( $\mu=10.6$ vs 4.7$)$.
Table 4 BV associated organisms

\begin{tabular}{lll}
\hline Organism & Detected & Not detected \\
\hline Lactobacillus iners & 152 & 118 \\
Gardnerella vaginalis & 106 & 164 \\
Lactobacillus crispatus & 90 & 180 \\
Atopobium vaginae & 66 & 204 \\
BVAB2 & 50 & 220 \\
Megasphaera spp. & 48 & 222 \\
Prevotella bivia & 44 & 226 \\
Sneathia sanguinegens & 39 & 231 \\
BVABI & 27 & 243 \\
BVAB3 & 16 & 254 \\
Mobiluncus curtisii & 5 & 265 \\
Mobiluncus mulieris & 4 & 266 \\
Bacteroides fragilis & 1 & 269 \\
\hline
\end{tabular}

Lactobacilli species tend to dominate the vagina of women without $\mathrm{BV} \cdot{ }^{28} \mathrm{An}$ important component of a healthy vaginal flora is Lactobacillus crispatus. ${ }^{26}$ It is hypothesized that during the development of $\mathrm{BV}$, the healthy Lactobacilli species, such as $L$. crispatus are displaced by other potentially more pathogenic agents. L. crispatus was found in $50 \%$ of our cases (Table 4). Unlike G. vaginalis, the presence of Lactobacillus crispatus was associated with a lower number of detected bacterial genera $(\mu=4.07$ vs 7.0$)$ (Figure 1B). Other Lactobacilli species are also components of the vaginal flora such as L. jensenii, L. gasseria, L. helveticus, L. gasseri and $L$. vaginalis. ${ }^{29}$ They produce hydrogen peroxide and lactic acid which are responsible for maintenance of an acidic environment and prevent colonization by some pathogens. ${ }^{30}$ Our results showed that when Gardnerella vaginalis was present, a lower proportion of healthy lactobacilli species were found indicating that healthy bacteria were lost as Gardnerella vaginalis colonized (Figure 2A). The presence of Lactobacillus crispatus was associated with a higher proportion of healthy lactobacilli species (60\% vs 30\%) (Figure 2B).

Other bacterial genera have been found in patients with bacterial vaginosis such as Megasphera, Atopobium, Sneathia, Prevotella, Bacteroides, Mobiluncus, Urea plasma and Mycoplasma. Our results showed a larger proportion of these BV-associated bacteria were found in samples containing G. vaginalis $\left(X^{2}=179.24, \mathrm{df}=1\right.$, p-value $<0.0001$ ). Nearly $33 \%$ of the total flora was composed of pathogenic agents when $G$. vaginalis was present compared to only $3 \%$ when absent (Figure 3A). The presence of Lactobacillus crispatus was associated with a lower proportion of reported BV associated organisms ( $<5 \%$ vs $21.4 \%$ ) (Figure $3 \mathrm{~B})$.

A benefit of the next generation sequencing approach was the ability to quantify proportions of bacterial reads and compare samples to one another. A plot of the bacterial composition was generated 
for each participant. For this analysis, all Lactobacilli species were combined into a single group and organisms that are not typically associated with BV were grouped into other species (Figure 4). We found that in cases with a lower proportion of $L$. crispatus and other Lactobacilli species, there was a higher proportion of G. vaginalis and other potentially pathogenic species. Only a small number of patients (14) were colonized with a majority of $G$. vaginalis (ie. greater than $50 \%$ ). The majority of the participants that tested positive for $G$. vaginalis also had other BV-associated organisms. There was a relatively high frequency of cases $(\mathrm{n}=77)$ with a high proportion of other organisms not traditionally associated with BV; such as Enterococcus, Staphylococcus, Streptococcus, Clostridium and Aurantimonas. The mean number of bacterial genera detected within the other organism dominated group was 10.2. The high bacterial diversity and absence of healthy, protective Lactobacilli species was suggestive of bacterial vaginosis.

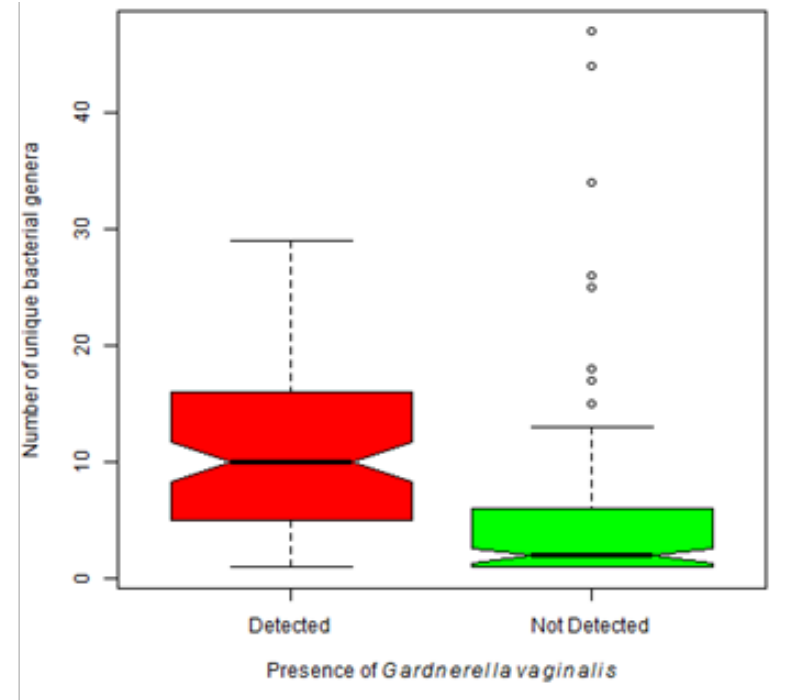

(A)

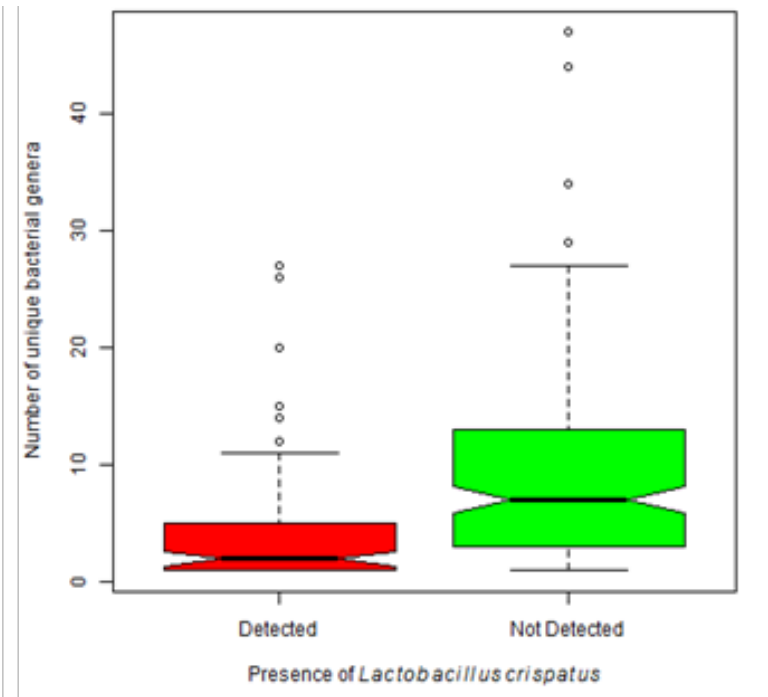

(B)

Figure I Number of bacterial genera detected varies depending upon organisms present.

A. Gardnerella vaginalis is found most often when accompanied by an environment that contains a higher bacterial biodiversity. That is the number of detected bacterial genera significantly differs between patients with Gardnerella vaginalis and those that do not (Kruskal-Wallis $X^{2}=73.45, d f=I, p-v a l u e<0.000 I$ ).

B. Vaginal samples colonized with Lactobacillus crispatusare associated with a lower bacterial biodiversity than those that do not contain this organism (Kruskal-Wallis $X^{2}=35.40, \mathrm{df}=\mathrm{I}$, $\mathrm{p}$-value $<0.000 \mathrm{I}$ ).

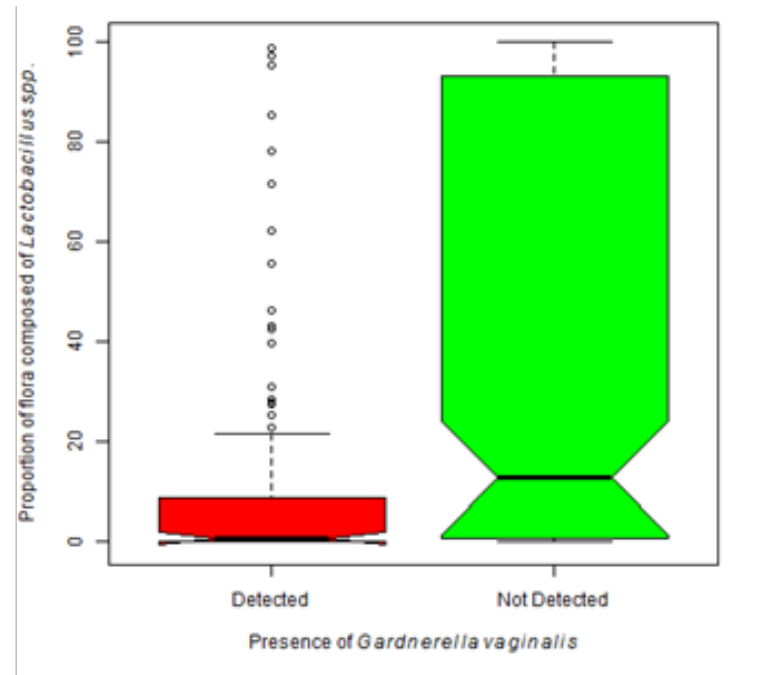

(A)

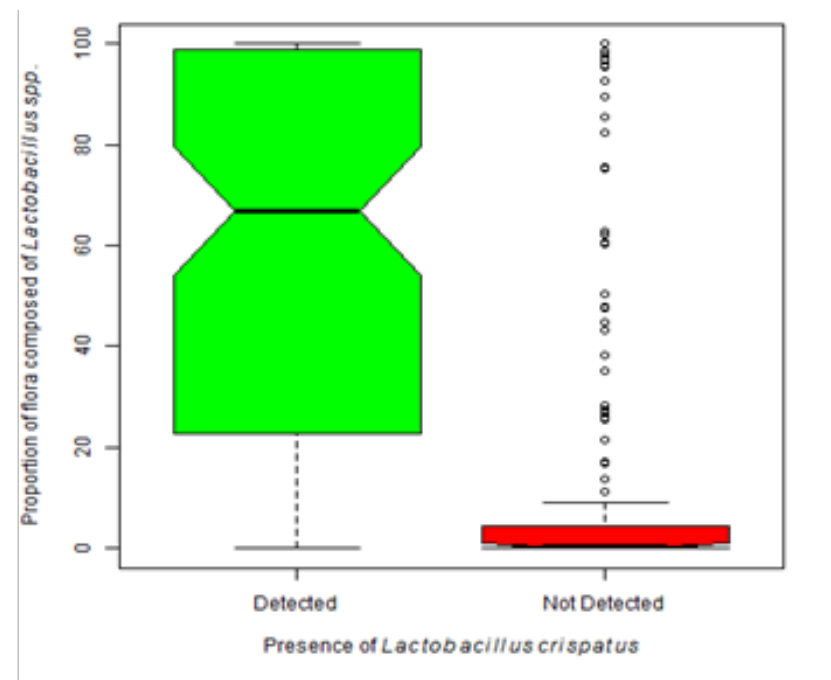

(B)

Figure 2 Healthy Lactobacillus species vary depending upon floral composition.

A. The presence of Gardnerella vaginalis is associated with a lower proportion of Lactobacilli species excluding L. iners. The proportion of Lactobacilli species significantly differs with the presence of $G$. vaginalis (Kruskal-Wallis $X^{2}=26.29, \mathrm{df}=I, p$-value $<0.000 \mathrm{I}$ ).

B. Dominance by one or more Lactobacilli species is considered normal. The presence of Lactobacillus crispatus is often described as protective. Our analysis shows that when Lactobacillus crispatus is present, the proportion of flora dominated by Lactobacillus species is significantly greater than when L. crispatus is not present (Kruskal-Wallis $\mathrm{X}^{2}=107.39, \mathrm{df}=\mathrm{I}$, $\mathrm{p}$-value $<0.000 \mathrm{I}$ ). 


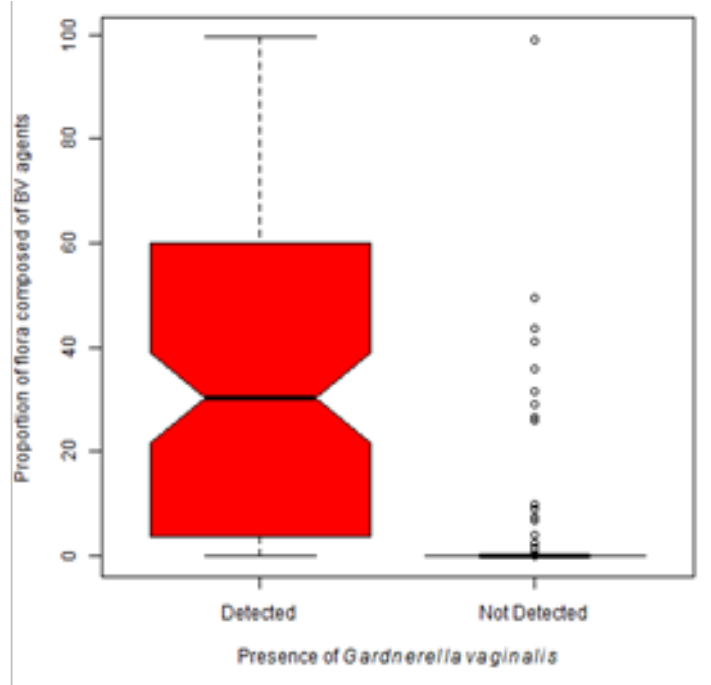

(A)

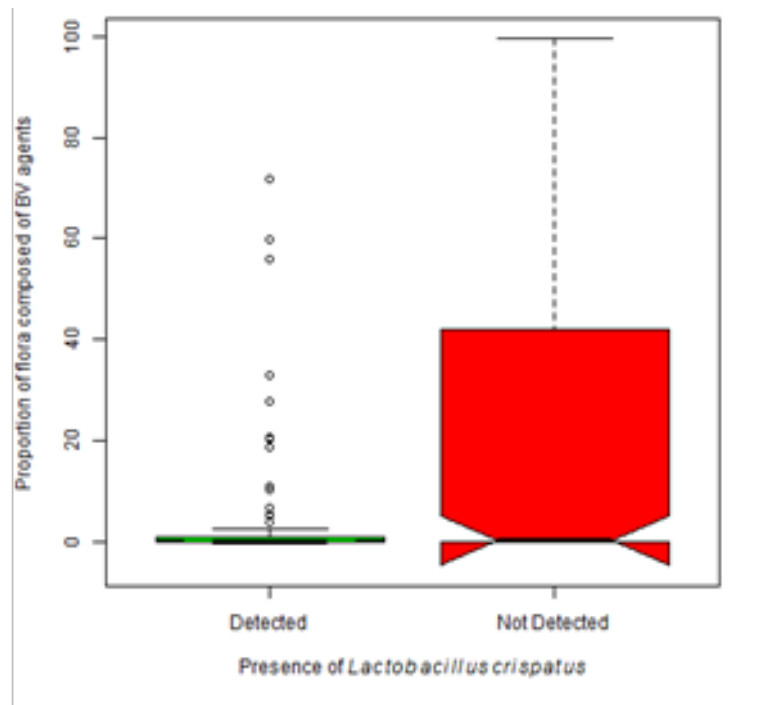

(B)

Figure 3 Proportion of pathogens varies with Gardnerella vaginalis and Lactobacillus crispatus.

A. The presence of Gardnerella vaginalis is most often associated with a flora dominated by species associated with the development of bacteria vaginosis. The proportion of flora occupied by BV associated organisms significantly differs between an environment colonized with $G$. vaginalis and an environment free from $G$. vaginalis (Kruskal-Wallis $\mathrm{X}^{2}=\mathrm{I} 79.24, \mathrm{df}=\mathrm{I}$, $\mathrm{p}$-value $<0.000 \mathrm{I}$ ).

B. An absence of Lactobacillus crispatus is associated with a flora dominated by BV associated organisms. The proportion of flora occupied by associated bacteria is significantly lower in women colonized with L. crispatus (Kruskal-Wallis $X^{2}=20.95, d f=I, p-v a l u e<0.000 I$ ).

In (Figure 4), all Lactobacilli species were grouped into a single grouping. It is known that not all Lactobacilli offer the same protection as L. crispatus. Where L. crispatus is considered protective because of its ability to produce hydrogen peroxide, most strains of L. iners are non- $\mathrm{H}_{2} \mathrm{O}_{2}$ producers, thus they are less protective than other Lactobacilli strains. ${ }^{31}$ Lactobacillus iners seems to be capable of surviving in diverse vaginal environments and in the face of multiple bacterial communities. ${ }^{26,32}$ These bacteria were found more often than any other organism in our study (Table 4). We found Lactobacillus iners in the presence of Lactobacillus crispatus and Gardnerella vaginalis (Figure 5A). Even though it can be found in the context of a normal flora, statistically it is more often found in higher proportions in the presence of Gardnerella vaginalis $\left(\mathrm{X}^{2}=7.83, \mathrm{df}=1\right.$, $\mathrm{p}$-value $=0.005$ ). There was not a statistically significant difference in the proportion of $L$. iners found in patients colonized with $L$. crispatus as compared to those that are not (Figure 5B). L. iners may be found in a healthy, asymptomatic woman, or in a patient showing clear symptoms of vaginosis. ${ }^{33}$ Treatment of $\mathrm{BV}$ with metronidazole causes the proportion of $L$. iners to increase without a subsequent increase in L. crispatus. ${ }^{34}$

Not all Lactobacilli species are equal, L. crispatus likely serves a protective role as it is a strong peroxide producer. ${ }^{31}$ The majority of women are colonized by more than one species of Lactobacilli. A second bacterial composition plot was generated that broke Lactobacilli into three groups; L. crispatus, L. iners and other hydrogen producing Lactobacillus species (Figure 6). In this figure, there exist three distinct groups that segregate largely based on Lactobacilli. Group 1 participants possessed a flora dominated by healthy Lactobacilli species and the majority of these participants were colonized with $L$. crispatus. As the proportion of hydrogen producing Lactobacilli decrease, the proportion of L. iners increased. Group 2 was $L$. iners dominant. There were a total of 152 participants that were colonized with some degree of $L$. iners. Of those participants, $50 \%$ were colonized with $G$. vaginalis. The flora of these participants was likely a transitional/ intermediate flora. ${ }^{35}$ The third group of participants was more likely to show symptoms of BV based on the microbial composition. The majority of this group was colonized with some degree of $G$. vaginalis.

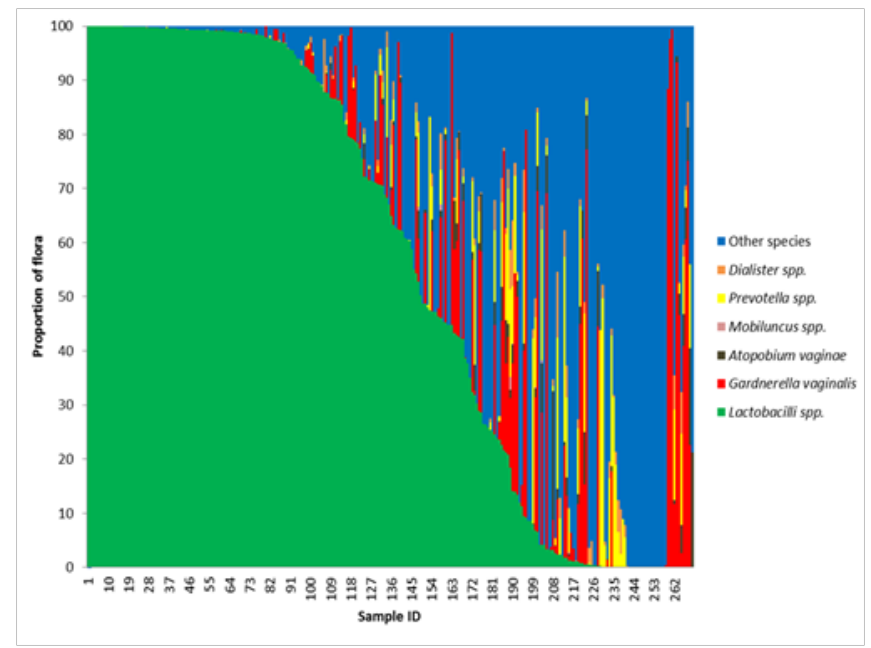

Figure 4 Loss of Lactobacilli species gives rise to non-resident bacteria.

The frequency of all Lactobacillus species, Gardnerella vaginalis, Atopobium vaginae, Megasphera species, Dialister species, Prevotella species were calculated for each participant in the study by dividing the sum of the reads for that organism by the total read count mapped to bacteria. If an organism was discovered that did not fit in the above referenced organisms, it was binned as other. A stacked bar graph was generated after sorting the participants by the frequency of Lactobacillus. As the frequency of Lactobacillus decreases, the frequency of the potential BV pathogens increases. 
(A)

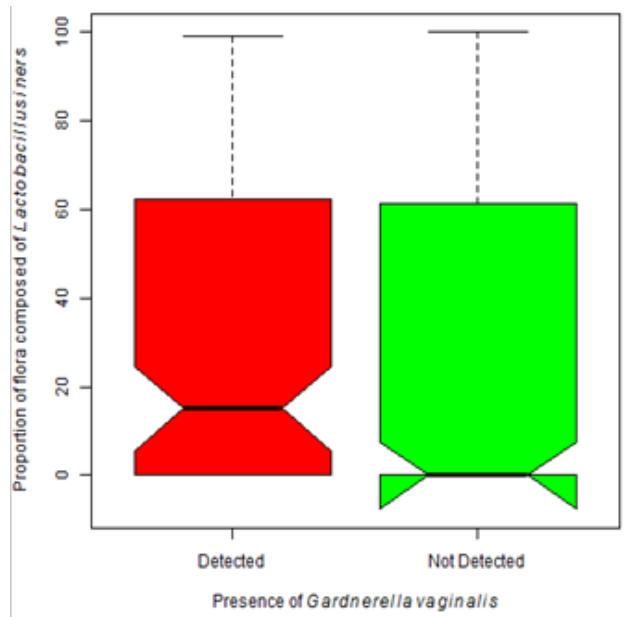

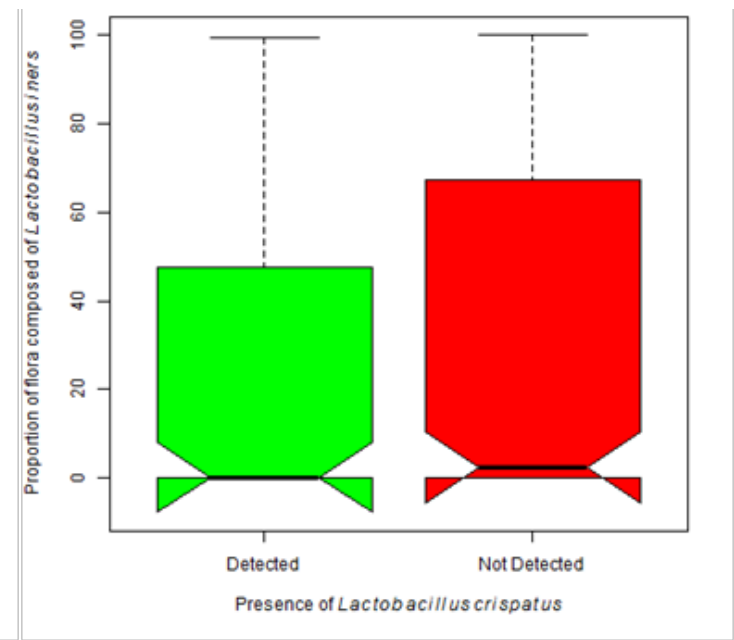

(B)

Figure 5 Colonization of Lactobacillus iners is more associated with Gardnerella vaginalis.

A. The presence of Gardnerella vaginalis is associated with a higher proportion of colonization by Lactobacillus iners (Kruskal-Wallis $X^{2}=7.83, d f=I, p-v a l u e=0.005$ ).

B. The presence of Lactobacillus iners does not statistically differ with the presence of Lactobacillus crispatus (Kruskal-Wallis $X^{2}=2.20, d f=I, p-v a l u e=0.138$ ).

The results of this study showed that BV was likely present when there were few healthy Lactobacilli species present to protect the vagina from non-resident microorganisms and the sum proportion of non-resident species was greater than the proportion of $L$. iners. Of the 270 participants in this study, 105 participants were predicted to be $\mathrm{BV}$ positive and the remainder predicted as $\mathrm{BV}$ negative. The three most commonly found organisms in samples predicted to be BV positive were Gardnerella vaginalis, Lactobacillus iners and Atopobium vaginae (Table 5). Over $60 \%(\mathrm{n}=65)$ of participants predicted BV positive were infected with Gardnerella vaginalis and Atopobium vaginae. Other studies have also shown that Atopobium vaginae rarely occurs in isolation, it is nearly always found in association with Gardnerella. ${ }^{36}$ Co-infection with $G$. vaginalis and $A$. vaginae is more likely to occur in women with recurrent $\mathrm{BV}$ infection. G. vaginalis was found in approximately $10 \%$ of women that were likely not suffering from BV symptoms. The majority of their bacterial flora was composed of healthy Lactobacilli (Figure 6). Organisms commonly associated with BV are rarely found in patients predicted to $\mathrm{BV}$ negative. ${ }^{37}$

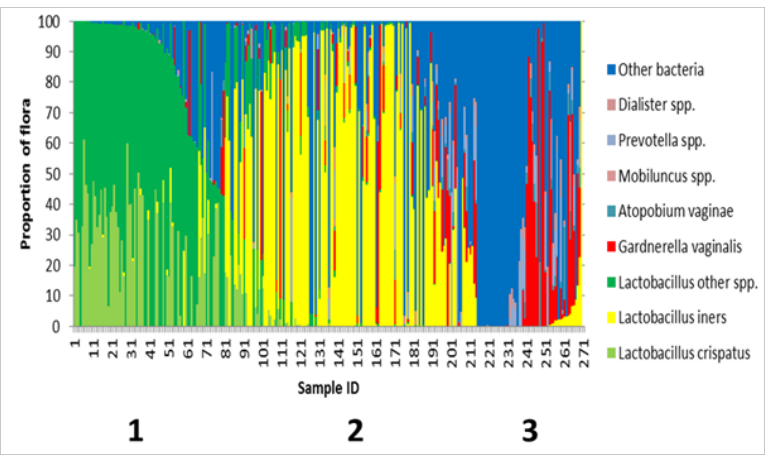

Figure 6 Speciation of Lactobacilli reveals a transitional flora.

The frequency of all Lactobacillus crispatus, Lactobacillus iners, and other hydrogen peroxide producing Lactobacillus species, Gardnerella vaginalis, Atopobium vaginae, Megasphera species, Dialister species and Prevotella species were calculated for each participant in the study by dividing the sum of the reads for that organism by the total read count mapped to bacteria. If an organism was discovered that did not fit in the above referenced organisms, it was binned as other. After sorting based on the proportion of Lactobacillus species, participants segregated into three distinct groups.
Table 5 Comparison of organisms after applying model for BV prediction

\begin{tabular}{lll}
\hline Organism & BV positive & BV negative \\
\hline Lactobacillus iners & 67 & 85 \\
Gardnerella vaginalis & 87 & 19 \\
Lactobacillus crispatus & 12 & 78 \\
Atopobium vaginae & 65 & 1 \\
BVAB2 & 50 & 0 \\
Megasphaera spp. & 48 & 0 \\
Prevotella bivia & 39 & 5 \\
Sneathia sanguinegens & 39 & 0 \\
BVABI & 27 & 0 \\
BVAB2 & 16 & 0 \\
Mobiluncus curtisii & 5 & 0 \\
Mobiluncus mulieris & 4 & 0 \\
Bacteroides fragilis & 1 & 0 \\
\hline
\end{tabular}

\section{Conclusion}

This study is one of the first studies to bring next generation sequencing of vaginal samples to community practices across the United States. This study shows that an NGS based, metagenomic assay can be delivered to clinical practices across the United States and deliver results within a time frame that can guide clinical treatment. This approach is superior to other diagnostic assays that often only look for the presence of single bacteria like Gardnerella vaginalis. NGS assays provide more comprehensive data that reveal a more complete picture of the distribution of bacteria giving clinicians a more complete understanding of their patient's vaginal flora. This approach is unbiased and does not rely on the incomplete knowledge of the etiology of BV. Our results showed that there existed three vaginal profiles that largely segregate based on the presence of facultative anaerobic organisms and the absence of healthy Lactobacilli. The first group was largely dominated by Lactobacilli species and was relatively 
homogenous. The second group was dominated by Lactobacillus iners and was more closely related to $\mathrm{BV}$ than the homogenous flora of group 1. Conversely, the flora of the third group was not dominated by any single organism but rather contained a mixture of organisms.

\section{Acknowledgements}

The authors of this manuscript acknowledge the efforts of all clinicians that referred participants for this study. We wish to thank Dr. Courtney A. Hunt for her efforts and her gracious review of the manuscript. We also think all employees of AIBioTech for their work on development of this project.

\section{Conflict of interest}

The authors of this study are employed by American International Biotechnology, which is a comprehensive contract research organization that offers clinical next generation sequencing services.

\section{References}

1. Pflughoeft KJ, Versalovic J. Human microbiome in health and disease. Annual review of pathology. 2012;7:99-122.

2. Human Microbiome Project Consortium. Structure, function and diversity of the healthy human microbiome. Nature. 2012;486(7402):207-214.

3. Boris S, Barbes C. Role played by lactobacilli in controlling the population of vaginal pathogens. Microbes Infect. 2000;2(5):543-546.

4. Hill GB. The microbiology of bacterial vaginosis. Am J Obstet Gynecol. 1993;169(2 Pt 2):450-454

5. Eschenbach DA, Davick PR, Williams BL, et al. Prevalence of hydrogen peroxide-producing Lactobacillus species in normal women and women with bacterial vaginosis. J Clin Microbiol. 1989;27(2):251-256.

6. Klebanoff MA, Turner AN. Bacterial vaginosis and season, a proxy for vitamin D status. Sex Transm Dis. 2014;41(5):295-299.

7. Allsworth JE, Peipert JF. Prevalence of bacterial vaginosis: 2001-2004 National Health and Nutrition Examination Survey data. Obstet Gynecol. 2007;109(1):114-120.

8. Hoffmann JN, You HM, Hedberg EC, et al. Prevalence of bacterial vaginosis and Candida among postmenopausal women in the United States. J Gerontol B Psychol Sci Soc Sci. 2014;69(Suppl 2):S205-S214.

9. Taylor BD, Darville T, Haggerty CL. Does bacterial vaginosis cause pelvic inflammatory disease? Sex Transm Dis. 2013;40(2):117-122.

10. Smart S, Singal A, Mindel A. Social and sexual risk factors for bacterial vaginosis. Sex Transm Infect. 2004;80(1):58-62.

11. Swidsinski A, Loening-Baucke V, Swidsinski S, et al. Polymicrobial Gardnerella biofilm resists repeated intravaginal antiseptic treatment in a subset of women with bacterial vaginosis: a preliminary report. Arch Gynecol Obstet. 2014;291(3):605-609.

12. Parma M, Stella Vanni V, Bertini M, et al. Probiotics in the prevention of recurrences of bacterial vaginosis. Altern Ther Health Med. 2014;20(Suppl 1):52-57.

13. Mitchell C, Manhart LE, Thomas K, et al. Behavioral predictors of colonization with Lactobacillus crispatus or Lactobacillus jensenii after treatment for bacterial vaginosis: a cohort study. Infectious Diseases in Obstetrics and Gynecology. 2012;2012:706540.

14. Wilson J. Managing recurrent bacterial vaginosis. Sex Transm Infect 2004;80(1):8-11.

15. Fredricks DN, Fiedler TL, Marrazzo JM. Molecular identification of bacteria associated with bacterial vaginosis. $N$ Engl J Med. 2005;353(18):1899-1911.
16. Amsel R, Totten PA, Spiegel CA, et al. Nonspecific vaginitis. Diagnostic criteria and microbial and epidemiologic associations. Am J Med. $1983 ; 74(1): 14-22$

17. Nugent RP, Krohn MA, Hillier SL. Reliability of diagnosing bacterial vaginosis is improved by a standardized method of gram stain interpretation. J Clin Microbiol. 1991;29(2):297-301.

18. Srinivasan S, Fredricks DN. The human vaginal bacterial biota and bacterial vaginosis. Interdiscip Perspect Infect Dis. 2008;2008:750479.

19. Srinivasan S, Hoffman NG, Morgan MT, et al. Bacterial communities in women with bacterial vaginosis: high resolution phylogenetic analyses reveal relationships of microbiota to clinical criteria. PLoS One. 2012;7(6):e37818

20. Fettweis JM, Serrano MG, Sheth NU, et al. Species-level classification of the vaginal microbiome. BMC Genomics. 2012;13(Suppl 8):S17.

21. Salipante SJ, Sengupta DJ, Rosenthal C, et al. Rapid 16S rRNA nextgeneration sequencing of polymicrobial clinical samples for diagnosis of complex bacterial infections. PLoS One. 2013;8(5):e65226.

22. Briselden AM, Moncla BJ, Stevens CE, et al. Sialidases (neuraminidases) in bacterial vaginosis and bacterial vaginosis-associated microflora. $J$ Clin Microbiol. 1992;30(3):663-666.

23. Schwartz T, Kohnen W, Jansen B, et al. Detection of antibiotic-resistant bacteria and their resistance genes in wastewater, surface water, and drinking water biofilms. FEMS Microbiol Ecol. 2003;43(3):325-335.

24. Edgar RC. Search and clustering orders of magnitude faster than BLAST. Bioinformatics. 2010;26(19):2460-2461.

25. Ihaka R, Gentleman R. R: A Language for Data Analysis and Graphics. Journal of Computational and Graphical Statistics. 1996;5(3):299-314.

26. Fredricks DN, Fiedler TL, Thomas KK, et al. Targeted PCR for detection of vaginal bacteria associated with bacterial vaginosis. J Clin Microbiol. 2007;45(10):3270-3276

27. Gardner HL, Dukes CD. Haemophilus vaginalis vaginitis: a newly defined specific infection previously classified non-specific vaginitis. Am J Obstet Gynecol. 1955;69(5):962-976.

28. Hillier SL. Diagnostic microbiology of bacterial vaginosis. Am J Obstet Gynecol. 1993;169(2 Pt 2):455-459.

29. Antonio MA, Hawes SE, Hillier SL. The identification of vaginal Lactobacillus species and the demographic and microbiologic characteristics of women colonized by these species. $J$ Infect Dis. 1999;180(6):1950-1956

30. Atassi F, Servin AL. Individual and co-operative roles of lactic acid and hydrogen peroxide in the killing activity of enteric strain Lactobacillus johnsonii NCC933 and vaginal strain Lactobacillus gasseri KS120.1 against enteric, uropathogenic and vaginosis-associated pathogens. FEMS Microbiol Lett. 2010;304(1):29-38.

31. Antonio MA, Rabe LK, Hillier SL. Colonization of the rectum by Lactobacillus species and decreased risk of bacterial vaginosis. J Infect Dis. 2005;192(3):394-398.

32. Jakobsson T, Forsum U. Lactobacillus iners: a marker of changes in the vaginal flora? Journal of Clinical Microbiology. 2007;45(9):3145.

33. Macklaim JM, Gloor GB, Anukam KC, et al. At the crossroads of vaginal health and disease, the genome sequence of Lactobacillus iners AB-1. Proc Natl Acad Sci USA. 2011;108(Suppl 1):4688-4695.

34. Hummelen R, Fernandes AD, Macklaim JM, et al. Deep sequencing of the vaginal microbiota of women with HIV. PLoS One. 2010;5(8):e12078.

35. Hillier SL, Krohn MA, Nugent RP, et al. Characteristics of three vaginal flora patterns assessed by gram stain among pregnant women. Vaginal Infections and Prematurity Study Group. Am J Obstet Gynecol. 1992;166(3):938-944 
36. Bradshaw CS, Tabrizi SN, Fairley CK, et al. The association of Atopobium vaginae and Gardnerella vaginalis with bacterial vaginosis and recurrence after oral metronidazole therapy. $J$ Infect Dis. 2006;194(6):828-836.
37. Potter J. Should sexual partners of women with bacterial vaginosis receive treatment? Br J Gen Pract. 1999;49(448):913-918. 